\title{
The kinematics of evolved planetary nebulae
}

\section{E. Margarita Pereyra, Michael G. Richer and Jose-Alberto López}

\author{
Instituto de Astronomía, Universidad Nacional Autónoma de México \\ Campus Ensenada, C.P. 22800, Ensenada B.C., México \\ email: mally, richer, jal@astrosen.unam.mx
}

\begin{abstract}
We have selected a group of 85 evolved planetary nebulae to study their kinematic characteristic based on spatially resolved, long-slit echelle spectroscopy. The data have been drawn from the San Pedro Mártir Kinematic Catalogue of PNe. The aim is to characterize in detail the global kinematics of PNe at advanced stages of evolution with the largest sample of homogenous data used to date for this purpose. The results reveal groups that share kinematic and photo-ionization characteristics of the nebular shell at the different late stages under study. The expansion velocities are typically larger than seen in earlier evolutionary stages, with the largest velocities occurring in objects with very weak or absent [N II] $\lambda \lambda 6548,6584$ emission line. These results shall serve to be compared to predictions of hydrodynamical models.
\end{abstract}

Keywords. planetary nebulae: general, ISM: kinematics and dynamics, techniques: spectroscopic

\section{Overview}

In order to gain a better understanding of the evolution of the nebular shell of planetary nebulae (PNe) in advanced stages of evolution, we have analized the expansion velocity of a large sample $(85 \mathrm{PNe})$ of these objects drawn for the San Pedro Martir Kinematic Catalogue of Planetary Nebulae (López et al. (2012) and this proceedings). We present in this contribution the results for a subsample $(35 \mathrm{PNe})$ of them, for which we have adopted stellar parameters $\left(L / L_{\odot}\right.$ and $\left.T_{\text {eff }}\right)$ from Frew (2008) for the central star, that allow us to place them in a H-R diagram, using the evolutionay tracks from Vassiliadis \& Wood (1994). We found a clear deceleration of the nebular shell as the central star's luminosity decreases. The largest expansion velocity is reached near the turn off point of the evolutionary track, right before the central star's luminosity decreases (see Fig. 1), as predicted by hydrodynamical models. Once the central star's luminosity declines, the expansion velocity of the nebula also decreases. This result shows that the higher the excitation degree the higher the expansion velocity of the nebular shell before the turn off point (see Fig. 2).

\section{Conclusions}

Due to the sample size and homogeneity of our data this work provides the ideal oportunity to tie the theory to observations from a solid statistical basis. The present results yield firm quantitative values to be compared with hydrodynamical models. Full results of this work will soon be published elsewhere.

\section{Acknowledgements}

The authors gratefully acknowledge financial support from CONACYT(82066) and UNAM-PAPIIT (IN110011) grants. E.M.P. is also grateful to the Dirección General de 


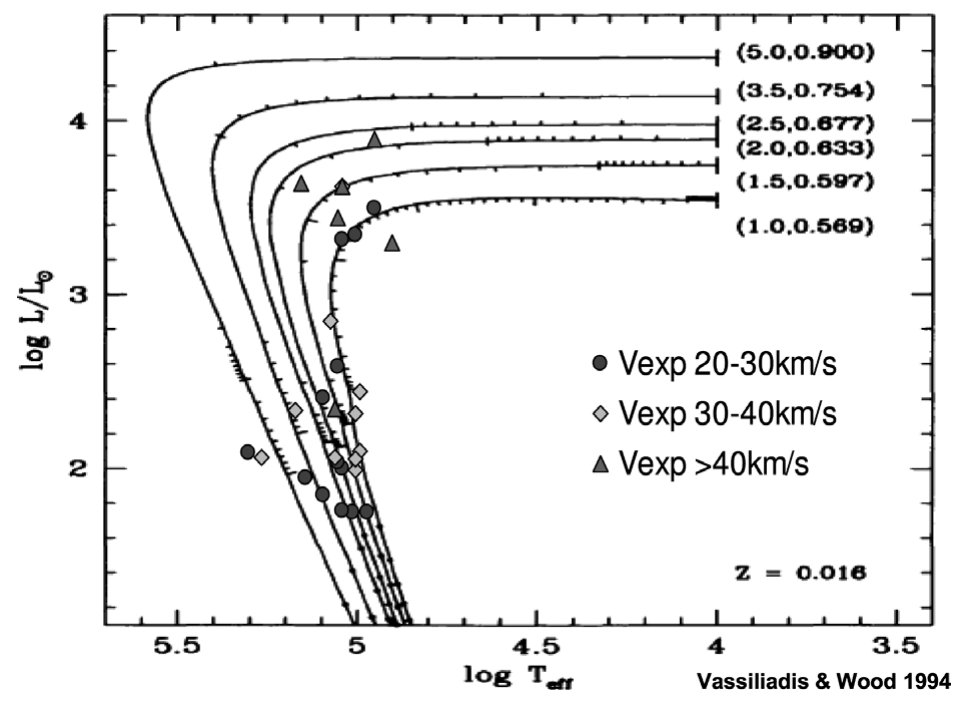

Figure 1. Velocity evolution of nebular shell in late stages.

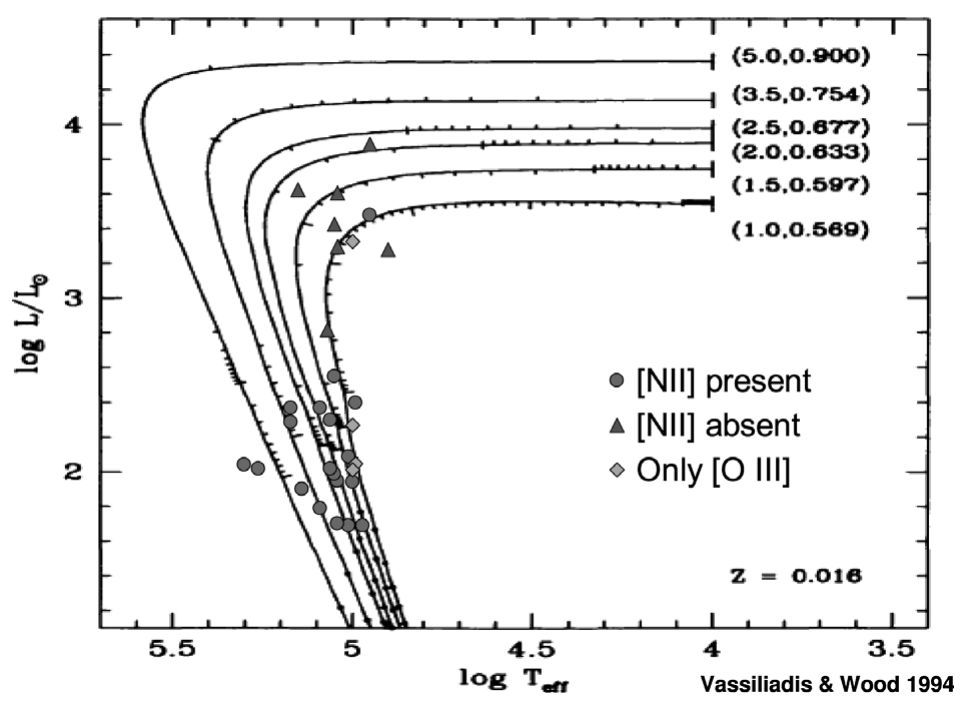

Figure 2. Excitation degree and kinematics are also linked: Objects with higher excitation show higher expansion velocities and weak or absent [NII] $\lambda \lambda 6548,6584$ emission lines.

Estudios de Posgrado de la UNAM and the LOC of IAU 283 for additional financial assistence.

\section{References}

Vassiliadis, E. \& Wood, P. R. 1994, ApJS 92, 125

Frew, D. J. 2008, PhD Thesis, Macquarie University

López, J. A., Richer, M. G., García-Díaz, Ma. T., Clark, D. M., Meaburn, J., Riesgo, H., Steffen, W., \& Lloyd, M. 2012, RMxAA, 48, 3 